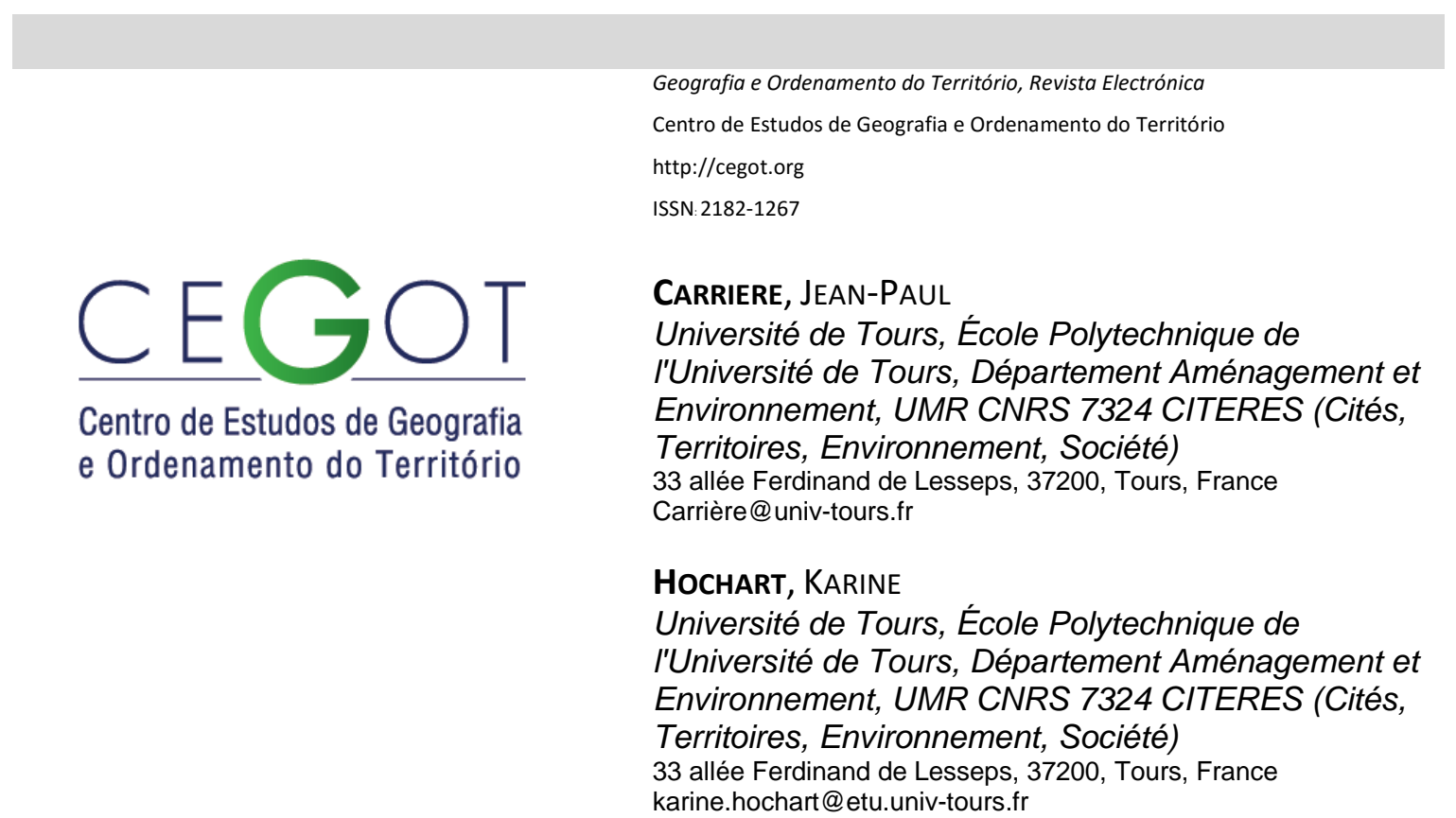

\title{
Un développement urbain durable et socialement inclusif est-il possible dans le contexte de fragmentation socio-spatiale des métropoles des pays émergents? Réflexions à partir des cas de Recife (Brésil) et de Chennai (Inde)
}

\begin{abstract}
É possível um desenvolvimento urbano sustentável e socialmente inclusivo no contexto de fragmentação sócio-espacial das cidades em países emergentes? Reflexões a partir dos casos de Recife (Brasil) e Chennai (Índia)

Is a sustainable and socially inclusive urban development possible in the socio-spatial fragmented context of cities in emerging countries? Reflections from the case of Recife (Brazil) and Chennai (India)
\end{abstract}

Referência: Carrière, Jean-Paul; Hochart, Karine (2016). Un développement urbain durable et socialement inclusif est-il possible dans le contexte de fragmentation socio-spatiale des métropoles des pays émergents? Réflexions à partir des cas de Recife (Brésil) et de Chennai (Inde). Revista de Geografia e Ordenamento do Território (GOT), n.o 9 (junho). Centro de Estudos de Geografia e Ordenamento do Território, p. 35-63, dx.doi.org/10.17127/got/2016.9.003

\section{RESUME}

Dans un contexte globalisé, le développement de villes socialement durables et inclusives implique de nombreux défis contradictoires. L'analyse comparée des cas de Chennai, en Inde, et de Recife, au Brésil, montre qu'au-delà des spécificités de ces métropoles, nous retrouvons des processus de fragmentation socio-spatiale comparables et un réel décalage entre un discours sur l'insertion sociale et des pratiques urbanistiques orientées par la quête d'attractivité, qui se soldent par l'accentuation des inégalités. Ces contradictions 
renvoient à des conflits entre planification stratégique, jeu des forces des marchés immobilier et foncier, et attentes des populations. L'analyse de ces contradictions invite à repenser l'articulation de la dialectique local/global et à s'interroger sur l'effectivité du " droit à la ville » dans les sociétés de pays émergents. La comparaison développée dans cet article s'appuie sur des recherches menées à Recife dans un premier temps au début de la décennie en cours, puis plus récemment à Chennai.

Mots-clefs: fragmentation socio-spatiale, habitat précaire, droit à la ville, attractivité, globalisation

\section{RESUMO}

Num contexto globalizado, o desenvolvimento de cidades sustentáveis e socialmente inclusivas envolve muitos desafios contraditórios. A análise comparativa dos casos de Chennai, na Índia, e de Recife, no Brasil, mostra que além das especificidades destas cidades, encontramos um processo de fragmentação sócio-espacial comparável e um hiato entre um discurso sobre a inserção social e práticas urbanisticas guiadas pela busca de atratividade, que resulta num agravamento das desigualdades. Essas contradições referem-se aos conflitos entre o planejamento estratégico, as forças dos mercados immobiliário e fundiário e as expectativas das populações. A análise dessas contradições sugere de repensar a articulação da dialética local / global e de questionar a efectividade do "direito à cidade" em sociedades dos países emergentes. A comparação proposta nesse artigo é baseada sobre pesquisas realizadas no Recife no inicio dos anos 2010, e mais recentamente em Chennai.

Palavras-chave: fragmentação sócio-espacial, assentamentos precários, direito à cidade, atratividade, globalização

\section{ABSTRACT}

In a globalized context, the development of sustainable and socially inclusive cities involves many competing challenges. The comparative analysis of the cases of Chennai, in India, and Recife, in Brazil, shows that beyond the specificities of these cities, comparable socio-spatial fragmentation process can be highlighted, as well as a gap between a discourse on social integration and urban practices guided by the pursuit of attractiveness, leading to an accentuation of inequalities. These contradictions refer to conflicts between strategic planning, forces of the real estate market, expectations of people. Thus, the analysis of these contradictions calls to rethink the articulation of the local / global dialectic and to question the effectivity of the "right to the city" in societies of emerging countries.

The comparison introduced in this paper is based on researches done first in Recife for the beginning of the actual decade, and more recently in Chennai.

Keywords: socio-spatial fragmentation, precarious settlements, right to the city, attractiveness, globalization 


\section{Introduction}

La recherche ${ }^{1} d^{\prime}$ un développement plus durable dans un monde global est à l'origine d'un ensemble de défis souvent contradictoires. Ainsi, en est-il des dynamiques de développement que connaissent nombre de grandes villes des pays émergents. Désireuses de se positionner sur le "marché mondial des territoires", ces grandes villes, à l'instar de celles du Brésil ou de I'Inde (Carrière et De la Mora, 2014, Hochart, 2014, Lucienne, 2014), connaissent des croissances très rapides, accompagnées de mutations sociétales importantes. Mais, elles restent marquées par un niveau de fragmentation socio-spatiale important, et d'inégalités toujours très fortes. La manifestation la plus tangible en est la coprésence au cœur-même des cités d'espaces de très grande précarité (les favelas au Brésil, et les slums en Inde) et de quartiers pleinement insérés dans la globalisation (quartiers d'affaires, condominiums fermés, etc.). Cette proximité géographique au sein des métropoles se matérialise par des effets de coupure urbaine et de segmentation du tissu urbain, qui en retour péjore l'attractivité des cités, à la fois sous l'effet de tensions urbaines exacerbées, de non-résolution de problèmes fonctionnels, et de dégradation d'image. Dès lors, les politiques urbaines restent marquées par des contradictions permanentes entre le souci de promouvoir des espaces attractifs et dotés d'une forte visibilité internationale et la volonté de réduire la fragmentation socio-spatiale par la résorption d'espaces de précarité.

Les croissances rapides que l'Inde et le Brésil ont connu ces dernières années a été un facteur d'accroissement des inégalités sociales, et à l'origine de profondes recompositions des espaces urbains et de nouvelles dynamiques métropolitaines. Dans cette perspective, nous confronterons deux cas de nature paradigmatique de métropoles en devenir, Recife, au Brésil, et Chennai, en Inde, toutes deux caractérisées par un degré toujours très élevé de fragmentation socio-spatiale et des politiques publiques locales de plus en plus marquées par le « paradigme de l'attractivité ».

\footnotetext{
${ }^{1}$ Cet article est issu d'une communication à l'occasion du colloque international "Proximités ", organisé à l'université de Tours, en mai 2015, sous le titre: La construction des proximités dans le contexte de fragmentation socio-spatiale des métropoles des pays émergents est-elle possible ? Réflexions à partir des cas de Recife (Brésil) et de Chennai (Inde).
} 


\section{La persistance de la fragmentation socio-spatiale : un défi pour les métropoles des pays émergents.}

Par "fragmentation socio-spatiale ", nous désignons un processus de "partition " ou d' "éclatement » de l'objet urbain. En réponse aux profondes mutations des métropoles brésiliennes liées à la mondialisation, la notion de fragmentation a été employée pour " décrire un nouveau type d'organisation socio-spatiale de la ville» (Vidal, 1994,p167). L'approche de Françoise Navez-Bouchanine (2002), bien que fondée sur d'autres réalités urbaines (notamment moyen-orientales), se transpose aisément à nos cas d'études en conjuguant fragmentations spatiale et sociale : « La fragmentation spatiale correspond à des situations urbaines caractérisées par un aspect éclaté, hétérogène et peu articulé physiquement et visuellement (discontinuité du tissu, hétérogénéité des paysages, des équipements et des services urbains, absence d'articulation entre les fragments). La fragmentation sociale, quant à elle, décrit l'émergence de territoires dans lesquels les populations vivent repliées sur elles-mêmes et où s'exprimerait l'absence de référence à la société globale.» (Navez-Bouchanine, cité par Chétry, 2010).

Saskia Sassen (1991) a été l'une des premières à lancer des pistes de réflexion pour saisir le lien entre la nouvelle organisation économique mondiale, née de la globalisation, et la production physique de la ville: avec le passage à une économie postfordiste, et les transformations du marché du travail liées à la mondialisation, une forme de dualisation de la société s'opère dans les villes, et singulièrement les plus grandes, ce qui favorise des logiques de séparation spatiale en leur sein. De la ville organique, on passe à la ville fragmentée tant physiquement que socialement. La réorganisation des structures sous l'effet de la globalisation entraîne alors de nouvelles connexions entre les espaces et les échelles qui impliquent de repenser la relation entre le local et mondial. En ce sens, le recours au concept de fragmentation suppose un changement de focale et d'angle d'observation. Ce concept permet alors une lecture du territoire à différentes échelles visant à comprendre les phénomènes qui se développent à chaque niveau territorial (Rojas, Vidal, 2002, p12) et contribue à alimenter des réflexions plus générales sur l'organisation des villes et leurs mutations. Son emploi participe ainsi à la rénovation du cadre théorique du débat urbain (Vidal, 1994, p171) et nous permet d'analyser les mutations s'opérant 
actuellement dans les métropoles du Sud, comme nous le verrons pour les cas de Recife et Chennai.

Recife, capitale de l'Etat du Pernambouc (cf. Carte $n^{\circ} 1$ ), $5^{\text {ème }}$ agglomération brésilienne de plus de 3 millions d'habitants (1 537704 pour le Municipe) prenant place, aux côtés de Salvador et de Fortaleza, parmi les principaux pôles constitutifs du niveau supérieur de l'armature urbaine du Nordeste, comme de celle de l'ensemble du pays, est à bien des égards représentative de la fragmentation des métropoles brésiliennes. En outre, comme le soulignent Maria Angela de Almeida Souza et Jan Bitoun (2015) si Recife a connu dans les années 2000 un dynamisme économique certain ${ }^{2}$, marqué par de nombreuses implantations industrielles et/ou immobilières nouvelles, l'agglomération $n^{\prime}$ a pas pour autant perdu le caractère de "métropole incomplète, périphérique et inégale", qui est le sien depuis de nombreuses années. En cela, ces auteurs s'inscrivent dans le droit fil de l'analyse de la métropolisation au Brésil, proposée par Milton Santos 30 ans plus tôt (M. Santos, 1982).

L'agglomération de Chennai, au $4^{\text {ème }}$ rang des métropoles indiennes avec près de 9 millions d'habitants (4 792949 pour la municipalité, en 2011) concentre les fonctions politiques, administratives et économiques de l'Etat du Tamil Nadu et constitue l'une des régions urbaines les plus dynamique du pays ${ }^{3}$ (Kennedy et al., 2014). Surnommée «Detroit de l'Inde » en référence à son rôle de plaque tournante de l'industrie automobile, Chennai est désormais un important centre d'investissement et un pôle attractif en termes d'emplois et de services ${ }^{4}$. Malgré ce véritable "boom économique » des deux dernières décennies, les emplois à Chennai reposent en majorité sur le secteur informel ${ }^{5}$, ce qui se traduit par une économie à faible revenu. Les répercussions sur les conditions de logement se manifestent par la persistance d'une grande précarité pour une part de la population chennaïte (Kennedy et al., 2014). La mise en perspective de ces deux cas nous permet d'étudier les recompositions spatiales métropolitaines qui se réalisent dans un contexte mondialisé, et les défis qui en résultent.

\footnotetext{
${ }^{2}$ Breno Bittencourt Santos $(2015$, p.350) indique que la RMR a vu sa contribution au PIB national passer de $1,48 \%$ en 2000 à $1,63 \%$ en 2010.

${ }^{3}$ Avec les districts de Tiruvallur et Kanchipuram

${ }^{4}$ Notamment services liés à la santé et à l'éducation

${ }^{5} 70 \%$ des emplois sont situés dans le secteur de l'économie informelle (Kennedy et al., 2014).
} 


\subsection{Recife, archétype de la métropole brésilienne fragmentée}

Recife, située sur un delta formé par 5 fleuves, forme avec ses voisines immédiates une vaste région urbaine jouant le rôle de métropole régionale, et étend son aire d'influence audelà des frontières de l'Etat de Pernambouc. Elle possède de nombreux atouts à faire valoir dans la concurrence métropolitaine, tant à l'échelle sud-américaine que mondiale : elle est le siège du plus grand parc technologique dédié aux NTIC du Brésil, le " Porto Digital ", et du second pôle médical du pays. Porte internationale du Nordeste, elle dispose d'un des principaux aéroports internationaux du pays. Dotée d'une puissante industrie du BTP, de secteurs de pointe (pharmaco-chimie, automobile...) elle dispose du grand complexe industrialo-portuaire de Suape qui abrite des raffineries de pétrole et le plus grand chantier naval de l'hémisphère sud. Au total, la Région Métropolitaine de Recife (RMR) occupe 3\% de la superficie de l'Etat du Pernambouc, mais concentre en 2010 plus de $42 \%$ de la population de cet Etat et produit plus de 50\% de son PIB (données IBGE, et De Almeida Souza et Bitoun, 2015)

Toutefois, la mise en valeur de ces atouts se heurte au morcellement institutionnel de l'espace métropolitain qui ne permet pas l'adoption de stratégies à la bonne échelle, en particulier lorsqu'il s'agit de trouver des réponses aux problèmes résultant de la fragmentation socio-spatiale, même si fut créée dès 1973 la Région métropolitaine de Recife (RMR) avec 14 autres municipes (Carrière, De la Mora, 2014).

En effet, la ville de Recife s'est construite selon un modèle d'occupation fragmentée, avec des morceaux de ville isolés, définis par des conditions d'habitabilité différenciées. Les contraintes topographiques du territoire et la reproduction de fortes inégalités persistantes au Brésil, en dépit de progrès indéniables en termes de réduction de la grande pauvreté au cours des 10 derrnières années ${ }^{6}$ y ont produit une grande dissémination de zones d'habitat

\footnotetext{
${ }^{6}$ Progrès qui, toutefois, se révèlent fragiles et fortement menacés par l'amplification de la crise sociale et économique que le Brésil connaît actuellement (en 2015/2016). De plus, le Brésil se situe toujours au 6ème rang mondial au vu de l'indice d'inégalité du PNUD (2011), malgré les évolutions positives de la décennie précédente (Dans le Nordeste, la proportion de ménages percevant un revenu inférieur au quart du revenu minimum a régressé de $43 \%$ à $23 \%$ entre 2000 et 2009 - de $23 \%$ à $11 \%$ au Brésil dans son ensemble - , tandis que le taux de mortalité infantile tombait de $26,5 \%$ à $15,6 \%$ - de $21,3 \%$ à $13,9 \%$ au Brésil - Sources IBGE in Neri et al. - 2012),). Le nombre de Brésiliens en situation de pauvreté extrême, (dont le revenu est inférieur à 70 Reais par mois, soit 25,5 Euros au cours de 2010), représente 8,5 \% de la population totale (plus de 16 millions). Au Nordeste, cette proportion monte à 18,5\%. Selon les statistiques de l'IBGE qui n'intègrent pas toutes les formes d'habitat précaire, on dénombre 6359 favelas dans tout le pays, localisées dans 323 des 5565 municipalités. On peut aussi parler d'une véritable polarisation de la précarité urbaine, puisque les 9 principales Régions Métropolitaines rassemblent à elles seules $78 \%$ des habitants vivant en favelas !
} 
précaire dans le tissu urbain. Mais, le processus d'urbanisation résulte autant de pratiques héritées d'une société intrinsèquement inégalitaire, que du fonctionnement du marché immobilier libéral aux caractéristiques sélectives et/ou des carences de la politique sociale.

Recife partage avec d'autres grandes villes du pays le fait d'être un lieu de condensation de phénomènes de grande pauvreté, d'exclusion et de violence, à des niveaux que peu de pays connaissent. Son Indice du Développement Humain est le plus faible de toutes les grandes métropoles millionnaires et il en est de même des indicateurs de bien-être (Bittencourt Santos, 2015). Elle occupe le second rang national en matière de criminalité.

Le développement spatial de la ville ne répond pas au schéma classique de l'opposition centre -périphérie. Le symbole le plus évident en est la faiblesse de la distance physique séparant les copropriétés fermées des "riches " et les ilots précaires des "pauvres ", ou favelas, voire leur voisinage direct. En dépit de l'amplitude des inégalités et de la distance sociale extrême qui séparent riches et pauvres, la proximité géographique des uns et des autres reste un des traits majeurs de la struture socio-spatiale recifense, ce que confirment toujours les études plus récentes (J. Bitoun, M.A. de Almeida Souza, 2015, p.26). C'est là I'héritage d'une production informelle qui a conduit les populations pauvres à occuper, soit individuellement, soit collectivement, des terrains aussi bien publics que privés (M.A. de Almeida Souza et al. 2015). Si effectivement, on peut observer une surconcentration des logements habités par des catégories "supérieures » ou "moyennes-supérieures » dans certains quartiers (tels que Boa Viagem en bord de mer, ou le quartier du Parque da Jacqueira, etc.) où domine la construction de très hautes tours de haut standing 7 par des sociétés de promotion immobilière généralement privées, il n'en reste pas moins que les logements occupés par des catégories "populaires ${ }^{8}$, très souvent construits en autoproduction clandestine et de façon informelle - $64,7 \%$ du parc total de logements de la RMR en $2010^{9}$ - se retrouvent dans tous les quartiers, y compris les plus centraux, en juxtaposition immédiate des immeubles les plus prestigieux. Or, tout indique que ce processus qui s'est accéléré au cours des 4 décennies écoulées se poursuit toujours. II a

\footnotetext{
${ }^{7}$ La législation en vigueur autorise des hauteurs de 40 étages

8 Une classification proposée par l'IBGE permet de distinguer 24 "catégories sociooccupationnelles" homogènes, à partir desquelles les travaux de l'Observatoire des métropoles obtiennent une typologie de 5 types d'habitat relativement homogènes, définis par leur mode d'occupation sociale : populaire-ouvrier, populaire-agricole, moyen, moyen-supérieur et supérieur.

${ }^{9}$ Selon la base de données de l'observatoire des métropoles élaborée à partir du recensement de 2010 (De Almeida Souza et al. 2015)
} 
représenté pendant cette période près de la moitié du stock de logements construits au sein de la RMR.

Comprendre les dynamiques urbaines au Brésil impose de se détacher d'une lecture par trop duale de l'espace urbain et nécessite de prendre en compte des phénomènes de discontinuité du tissu urbain, et d' " entre soi » qui caractérisent la composition sociale des quartiers. Recife se présente comme une sorte de patchwork, aux couleurs et aux formes contrastées, voire opposées (verticalité des quartiers riches vs horizontalité des favelas ${ }^{10}$, constructions de standing dans les zones accessibles, sans relief et non inondables vs étalement des favelas sur les pentes et les bords de rivière...), une collection de " morceaux de ville ", occupés alternativement par des populations aisées ou très aisées ou au contraire en grande précarité.

Certes, on ne peut assimiler les favelas ou les copropriétés fermées, les condominios, à de simples enclaves de pauvres, ou de riches, sans articulation avec l'ensemble de l'espace urbain (Chétry, 2013, Ninnin, 2014). La réalité favelada est plus hétérogène (Valladares, 2000, 2006) que l'image qui en est véhiculée par les medias; mais la persistance de très grandes différences dans les morphologies des espaces bâtis et de discontinuités brutales, qui se conjuguent avec des inégalités de conditions de vie criantes, nous conduisent à conserver le concept de fragmentation comme clé de lecture.

Recife, pour des raisons historiques (Carrière, De la Mora, 2014), se distingue par l'importance de son habitat précaire: en 2010, 852700 habitants de la Région Métropolitaine de Recife (RMR) vivaient dans des ilots précaires, soit $23,2 \%$ de la population totale (plus qu'à Rio de Janeiro -14,4\%- ou São Paulo-11\%-). Les familles pauvres y sont réparties sur tout le territoire, occupant en priorité les espaces délaissés pour des raisons topographiques (pentes, zones inondables...) ou géographiques (proximité de nuisances diverses...). Dans ces ilots éparpillés, vivent des populations sans emploi et sans revenus formels, dans des conditions de forte exclusion sociale ${ }^{11}$ (De la Mora et Souza, 2002). Le total des ilots résidentiels précaires recensés dans la RMR s'élève à 1046, dont 441

\footnotetext{
${ }^{10}$ Même si un processus de verticalisation et de densification commence à se dessiner dans les favelas (De Almeida Souza et al. 2015)

${ }^{11}$ Ce qui n'empêche pas pour autant la fréquentation par ces mêmes habitants d'équipements d'envergure, comme le Parc Dona Lindu, ou leur participation active à des évènements culturels majeurs comme le Carnaval. La situation des populations pauvres du Brésil s'analyse toujours difficilement en employant les catégories habituelles utilisées pour caractériser la pauvreté dans d'autres pays.
} 
à Recife-même et 111 à Olinda, la seconde ville de la RMR (Alves dos Santos. Barbosa Mahmood, 2013).

La distribution spatiale des zones d'habitat précaire à Recife « intra-muros » (cf. Carte $\left.n^{\circ} 4\right)$ illustre bien la dissémination des espaces de pauvreté dans l'ensemble du tissu urbain central et péricentral, ce qui reste l'un des traits dominants de la géographie sociale et du paysage urbain de la ville. Cette carte n'est pas à elle seule représentative de la fragmentation socio-spatiale, car il aurait fallu pour cela pouvoir y faire figurer d'autres informations concernant aussi bien les zones résidentielles des élites que les espaces commerciaux de haut standing ou la localisation des services supérieurs, mais elle permet néanmoins d'inférer la persistance des inégalités urbaines et l'existence d'un processus de fragmentation socio-spatiale qui débouche sur un modèle de division sociale de l'espace très éloigné de la simple opposition centre - périphérie, comme on la rencontre souvent en Europe et en France, plus particulièrement. A l'instar de plusieurs autres métropoles brésiliennes (Salvador, Belo Horizonte, Rio, etc.) la fragmentation socio-spatiale reflète les les profondes inégalités structurelles qui traversent la société, mais s'explique aussi par les dysfonctionnements majeurs d'un système de production du cadre bâti très largement soumis aux forces des marchés immobilier et foncier, en dépit d'importants programmes publics lancés récemment comme le programme Minha Casa, Minha Vida12. D’un côté, la

\footnotetext{
12 Depuis le début des années 2000, et surtout l'arrivée au pouvoir du Président Lula, en janvier 2003, les initiatives, locales ou fédérales, se sont multipliées en réponse au problème du " mal-logement ", et la production publique du logement, lato sensu, articulant toutes les interventions des différents niveaux de la Fédération brésilienne (Union Fédérales, Etats et Municipes) a connu un essor indéniable. Le statut de la ville, reconnaissant l'existence d'un «droit à la ville » pour tous, adopté à travers la loi de 2001 s'applique à l'échelle fédérale. Son adoption a été prolongée par la création, en 2003, d'un ministère fédéral des villes. Les Etats fédérés, à l'instar du Pernambouc, ont aussi lancé des politiques du logement spécifiques d'aménagement des favelas ou en appui de celles de l'Etat; et l'Etat du Pernambouc a lui-même mis en place un cadre normatif, avec la création des ZEIS et du PREZEIS, qui servira ultérieurement de référence à la politique urbaine nationale.

La volonté politique de se doter d'instruments de politique publique favorisant une meilleure inclusion sociale est réellement affichée depuis cette époque, mais sans pour autant toutefois porter vraiment atteinte aux forces de marché toujours orientées vers une production immobilière de standing. Le programme national le plus spectaculaire est le programme "Minha casa, Minha vida». Ce programme, institué par la loi n¹1.977 du 7 juillet 2009 est destiné à favoriser l'accès à la propriété des ménages modestes. L'objectif est de financer la construction de deux millions de logements en accession, dont $60 \%$ pour des familles ayant un revenu inférieur à 1395 Reais par mois. Même si les seuils de revenus exigés pour l'octroi des aides sont faibles, ces dernières inversement proportionnelles aux ressources des familles, ne sont pas pour autant accessibles à tous et ne permettent pas aux très pauvres de lever l'obstacle foncier. Si les effets en sont quantitativement significatifs, son impact social reste donc partiel. II convient aussi de mentionner les investissements en assainissement urbain, et "d'urbanisation des favelas" prévus dans le cadre du Programme (national) d'accélération de la croissance de 2007, qui ont permis des opérations ponctuelles d'amélioration des conditions de vie dans les favelas (aménagements de l'espace public, viabilisation de certains îlots...).
} 
grande faiblesse du marché locatif et l'action de la Caixa Fconômica Federal $(C E F)^{13}$ en matière d'habitat social, qui ne favorise que l'accession à la propriété par ses aides dans le cadre de financements dits "sociaux", auxquels les plus pauvres ne peuvent avoir accès, participent à l'exclusion de fait de ceux-ci du marché formel des logements; de l'autre, la promotion immobilière privée très active se concentre sur la production des "condominios", copropriétés fermées de haut et très haut standing réservées aux catégories sociales supérieures ou de la "nouvelle classe moyenne ». Compte tenu de l'organisation socio-spatiale évoquée ci-dessus, on comprend alors que l'indice du Bien-être urbain, calculé par les chercheurs du réseau de l'Observatoire des métropoles à partir des données du recensement de $2010^{14}$ montre qu'à l'exception de quelques zones restreintes du littoral sud de la ville (Boa Viagem), ou de quelques quartiers plus au nord (Casa Forte, Jacqueira, etc.), les niveaux de cet indice soient très faibles sur l'ensemble du territoire de la ville-centre comme de la RMR, témoignant ainsi d'une forte corrélation entre la typologie socio-spatiale des îlots et quartiers de la métropole et le niveau de précarité que reflète I'indice (B. Bittencourt Santos, 2015).

\subsection{Chennai, métropole indienne ségrégée et multipolaire}

Les dynamiques urbaines en Inde résonnent avec celles observées au Brésil, bien que s'opérant sous des modalités différentes. Le développement de Chennai questionne la centralité urbaine. En effet, Chennai, originellement nommée Madras, n'était à sa formation qu'un village situé sur la côte du Coromandel qui n'a cessé de croître et de s'étendre en

\footnotetext{
Au niveau local, les villes ne sont pas non plus totalement dépourvues d'instruments pour tenter de réduire la précarité urbaine. Elles peuvent notamment mobiliser leur budget participatif, directement inspiré de l'expérience de Porto Alegre. Concrètement, il s'agit à travers des réunions de quartiers et de " microrégions" (elles sont 18 au sein du Municipe de Recife) de définir des priorités d'aménagement local et la répartition des investissements publics dans la ville, plus particulièrement dans les quartiers où l'I.D.H. est faible, les citoyens étant in fine invités à classer ces priorités par vote électronique. Le budget participatif se veut donc à la fois un instrument d'inclusion sociale, et de gestion démocratique des politiques publiques requérant la participation des citoyens sans exclusive. A Recife, jusqu'en 2012, le budget participatif était le plus important du pays, selon le site web de la Municipalité.

Le lecteur pourra trouver une analyse historique beaucoup plus détaillée des politiques publiques de l'habitat menées depuis les années au Brésil et à Recife dans l'article de De Almeida Souza et al (2015,pp. 270 et suivantes).

${ }^{13}$ En charge de la collecte de l'épargne nationale, la CEF est la seconde institution financière publique d'Amérique latine.

${ }^{14}$ L'indice du Bien-être Urbain (IBEU) est un indice synthétique calculé à partir de variables multiples représentatives de la mobilité urbaine, de qualité de l'environnement physique, de la qualité de l'habitat, du degré de fourniture des services collectifs et de l'infrastructure urbaine.
} 
incorporant d'autres noyaux d'habitat (Muthiah, 2014). Cette particularité marque la structure actuelle de la ville : il n'existe pas de centre-ville, mais des centres qui sont le reflet de cette histoire (Oliveau, 2007).

L'organisation spatiale des villes et des villages en Inde reflète la hiérarchie de la société selon le système de castes, par essence ségrégatif. Si le territoire urbain conserve encore par endroits l'empreinte millénaire du système stratifié des castes ${ }^{15}$, il a néanmoins subi davantage l'influence de la colonisation britannique : de nouveaux quartiers se sont édifiés, en application d'autres principes ségrégatifs basés sur des déterminants socio-économiques (Vaguet, 1997). Originellement divisée entre "ville blanche ", lieu d'occupation des anglais, et "ville noire ", territoire occupé par les indiens, Chennai s'est construite sur un modèle dual, laissant aujourd'hui place à une forte hétérogénéité entre les quartiers. L'étalement urbain a suivi un mode classique, le long des axes majeurs de communication et contraint par la présence des cours d'eau (Oliveau, 2007). La ville s'est d'abord édifiée au nord avec l'avènement de l'industrie, puis dans sa partie sud, principalement résidentielle, avant de connaître une densification du tissu urbain à la fin du $19^{\mathrm{ème}}$ siècle. Après l'Indépendance, l'ordre spatial s'est entièrement remodelé, la morphologie polycentrique et la multiplication des aires résidentielles en périphérie complexifiant le schéma traditionnel. Le développement des transports individuels, l'insuffisance de l'offre de logements et la forte pression immobilière ont participé à l'important accroissement des marges extérieures de l'agglomération.

Ces transformations spatiales majeures sont restées longtemps incontrôlées. C'est seulement en 1974, pour mieux réguler cette croissance urbaine rapide, que l'autorité de développement de la Région métropolitaine de Madras $^{16}$ a été instituée et chargée d'élaborer un Master Plan à l'échelle de la métropole (cf. Carte n5). La Région métropolitaine reste l'autorité principale en matière d'aménagement territorial, mais la compétence de planification urbaine est partagée entre plusieurs opérateurs intervenant à

\footnotetext{
15 Certains quartiers historiquement occupés par une certaine communauté ont conservé cette caractéristique : à Chennai, les quartiers de Mylapore ou Triplicane par exemple, restent encore habités en majorité par des communautés brahmanes.

${ }^{16}$ MMDA, Madras Metropolitan Development Authority, a été constitué en 1972 à la suite du Tamil Nadu Town and Country Planning Act 1971 et est devenu un organisme officiel en 1974. L'agence est ensuite renommée Chennai Metropolitan Development Authority en 1996.
} 
différentes échelles, de celle de la ville à celle de l’Etat; cette multiplicité des acteurs créant de multiples problèmes de coordination. A l'instar de Recife, les reconfigurations urbaines et le morcellement des institutions à Chennai posent la question de la pertinence des instruments de gestion métropolitaine.

La nouvelle donne économique indienne des années 1990 a propulsé Chennai au rang de pôle financier et lieu d'investissement technologique, impactant considérablement le développement de la ville, notamment en contribuant à son expansion au sud et à l'ouest (Oliveau, 2007) et entraînant l'émergence de nouvelles formes de polarisation sur les espaces périurbains (Arabindoo, 2009). Cependant, les récentes mutations de la métropole n'ont pas résorbé les inégalités socio-spatiales : $23 \%$ de la population chenaiïte habite toujours dans les bidonvilles, disséminés dans le tissu urbain, et localisés principalement sur des espaces délaissés par la ville "formelle ", le long des cours d'eau ou des voies de transport (Kennedy et al. 2014). La ressemblance avec Recife est frappante, comme on peut l'observer en comparant les cartes de localisation des îlots précaires, $n^{\circ} 4$ et $n^{\circ} 6^{17}$.

Marquée par une forte multipolarité, la structure urbaine présente aujourd'hui des déséquilibres importants et l'ensemble reste globalement fracturé entre le nord et le sud : alors que la partie nord est densément occupée par les classes populaires et concentre une grande partie des habitats précaires, le sud de la ville, qui accueille les quartiers résidentiels des classes moyennes ainsi que les infrastructures métropolitaines, participe à construire l'image d'une ville moderne globale. Mais, au sein même de ces quartiers, on observe une juxtaposition d'îlots de précarité enclavés et d'espaces intégrés au développement économique. La coprésence de quartiers fortement contrastés caractérisés par des niveaux d'intégration différenciés est la manifestation la plus tangible de la fragmentation urbaine, observable dans les deux métropoles (cf. Photographies $n^{\circ} 1$ et $n^{\circ} 2$ ).

\footnotetext{
${ }^{17}$ La carte $n^{\circ} 6$ présente les mêmes limites d'interprétation que celles de la carte $n^{\circ} 3$ mentionnées plus haut dans le corps du texte.
} 


\section{Chennai et Recife : des processus de fragmentation socio- spatiale aux causes et aux formes comparables et des contradictions partagées}

Avec l'entrée des métropoles dans le mouvement de la globalisation, l'ensemble des structures, de la gouvernance, des processus décisionnels, des échelles et des logiques urbaines a été reconsidéré; de nouvelles dynamiques aux enjeux complexes se sont développées.

Ainsi, à Recife, les choix stratégiques d'aménagement sont de plus en plus influencés par le " paradigme de l'attractivité » et la recherche d'un changement d'image. Comme les autres grandes villes du Brésil, Recife est soucieuse de " se vendre " sur le marché global des territoires, d'adapter sa centralité aux exigences de la mondialisation par des aménagements appropriés (centres d'affaires, palais des congrès, équipements culturels et de loisir, hôtels de luxe, pôles de recherche, etc....). II s'agit de concentrer des fonctions supérieures de niveau international et de capter des flux d'investissement étrangers. La course à la verticalité, les grands " gestes architecturaux ", les opérations de marketing urbain, les grands événements emblématiques (Coupe du monde, ...) sont autant de manifestations de cette stratégie.

Dans cette perspective, la fragmentation urbaine y est souvent perçue comme un facteur de dégradation de l'image et de perte d'attractivité. II en résulte des tensions permanentes entre des politiques publiques visant à éradiquer des concentrations de pauvreté et des politiques menées sous la pression des mouvements sociaux en vue d'améliorer les conditions de vie locales dans les quartiers précaires. Recife n'échappe pas à ce mouvement contradictoire, avec d'un côté la création de Zones Spéciales d'Intérêt Social (ZEIS), destinées à maintenir sur place les quartiers précaires en améliorant leurs conditions d’habitabilité (comme on le verra plus loin), et, d'un autre côté, des projets menés avec le secteur privé visant à libérer le foncier occupé par l'habitat informel, en vue de créer des ensembles de prestige, au sein ou à proximité des favelas les plus importantes, telle que la favela du Coque au centre de l'agglomération (Lucienne, 2014). Situé sur un emplacement stratégique, à la croisée des flux de circulation, et sans avoir bénéficié d'une remise aux normes de ses logements et de ses infrastructures de base, ce quartier a cependant fait l'objet d'interventions lourdes, telles que la déviation du fleuve Capibaribe, la création 
d'une gare fluviale et d'une station de métro, la construction du forum de la justice, ou, sur ses franges, d'un gigantesque shopping center. Ces équipements ne sont pas sans effet positif sur les conditions de vie des habitants, mais relèvent d'une toute autre logique de renforcement de la centralité et de l'attractivité métropolitaine, sans contribuer réellement à l'amélioration de la qualité des logements et de l'espace public de la favela. Autre exemple, le projet Novo Recife, proposé par un consortium d'entreprises privées, prévoit la construction de 12 tours de 40 étages dans une zone fortement dégradée du Recife ancien, projet qui suscite en réaction un mouvement social de grande ampleur. On pourrait encore évoquer, mais cette fois-ci en périphérie, à São Lourenço da Mata, les aménagements associés à la construction du grand stade de football en vue de la coupe du monde, qui a induit le démantèlement de quartiers précaires et la délocalisation de leurs habitants.

Le secteur immobilier privé aujourd'hui dominé par des groupes puissants (Oderbrecht, etc...), voit dans la restructuration des espaces centraux dégradés, dans ou hors ZEIS, de nouvelles opportunités foncières pour de grands projets, peu compatibles avec le besoin d'inclusion sociale des populations en grande précarité.

Le niveau de fragmentation socio-spatiale et le développement en contiguïté de zones de pauvreté et d'îlots d'opulence, y compris au cœur du centre historique, est tel qu'une part importante de la population urbaine est toujours dans l'incapacité de faire valoir son « droit à la ville ", pourtant explicitement reconnu par la Constitution de 1988 et par la Loi Estatuto da Cidade de 2001 ; alors que dans le même temps la fonctionnalité et la compétitivité des quartiers ayant vocation à accueillir les activités mondialisées s'en trouvent affectées.

A Chennai, des constats du même ordre s'imposent. A la suite des réformes économiques libérales de 1991, les métropoles indiennes connaissent une série de bouleversements structurels liés aux stratégies de planification de plus en plus orientées par les lois du marché. La volonté affichée du gouvernement du Tamil Nadu de développer des secteurs d'activités spécifiques comme l'informatique et l'automobile a inscrit Chennai dans le mouvement de la globalisation. L'apport de capitaux étrangers associé à des politiques urbaines incitatives a alors conduit à la création de "corridors urbains » accueillant ces nouvelles activités économiques, des parcs industriels et des instituts d'enseignement et de recherche. Aménagé sur 45 kilomètres au sud de Chennai, I'IT corridor ${ }^{18}$ est un exemple

\footnotetext{
${ }^{18}$ L'IT corridor (Old Mahabalipuram Road) est l'une des principales routes de la périphérie sud de Chennai développée en 2008 pour accueillir de grandes sociétés de service et d'informatique. L'agence de
} 
particulièrement emblématique de cette orientation stratégique de la planification urbaine. En l'espace de dix ans, près d'une centaine de milliers d'emplois ont été créés dans le domaine des technologies de l'information, attirant une population plus qualifiée et plus exigeante en matière de qualité de vie (Kennedy et al. 2014). Kamala Marius-Gnanou (2010) montre que ce "processus de déconcentration et de redéploiement des fonctions, tant économiques que résidentielles, vers la périphérie " participe à la réorganisation de l'espace urbain, en produisant notamment une "spécialisation et une fragmentation fonctionnelles des territoires » (Marius Gnanou, 2010). Par ailleurs plusieurs mégaprojets d'infrastructures ont été récemment envisagés à Chennai, tels que la construction du port d'Ennore, l'autoroute surélevée de Maduravoyal à Koyambedu, conçue pour desservir le port, I'Outer Ring Road (ceinture périphérique) ou encore la construction du métro. Correspondant aux modèles internationaux d'infrastructures métropolitaines, ces projets visent à doter Chennai des attributs d'une "world class city" et à booster son développement économique, mais ils restent relativement peu adaptés aux conditions locales : ainsi, l'autoroute surélevée est spécialement conçue pour le transit de camions transportant des voitures destinées à l'exportation. Chennai semble en effet davantage planifiée pour « satisfaire les investisseurs étrangers plutôt que les résidents locaux. " (Saravanan, Jayaraman, 2011). Le plan de redéveloppement du quartier central populaire de Foreshore Estate $^{19}$, adopté en 2009, qui prévoit le relogement en périphérie d'une grande partie des habitants en vue de développer des quartiers résidentiels et hôtels de luxe, est un autre exemple qui traduit les priorités fixées par les autorités publiques en termes d'aménagement. Dans le rapport élaboré en 2011 lors de la campagne "Save Chennai beaches ", Saravanan et Nithyanand Jayaraman soulignent que ce projet n'est pas formulé isolement : mis en perspective avec les autres projets de renouvellement urbain, il apparaît une nette convergence entre les différents projets menés simultanément à l'échelle de la

développement des infrastructures routières, le TNRDC, en charge du projet, indique que le but est de " promouvoir le corridor comme une infrastructure de classe-mondiale en phase avec une image attractive et moderne du Tamil Nadu ». Le financement du projet s'est fait par le biais d'un partenariat avec une filiale spécialisée, l'IT Expressway Ltd' (ITEL), qui a servi d'outil de placement pour l'agence TNRDC (d'après le site du TNRDC, http://tnrdc.com/it-corridor-project/).

${ }^{19}$ Fore Shore Estate est un quartier populaire situé le long de la plage Marina. A l'origine village de pêcheurs constitué de huttes, le quartier a été progressivement incorporé à l'espace urbain et considéré comme " bidonville ". Dans les années 1960, le gouvernement a relogé certains habitants dans des bâtiments construits in-situ, avec des matériaux de mauvaise qualité menant à une détérioration rapide des conditions d'habitabilité de ces structures. Le plan de redéveloppement de Foreshore Estate prévoit d'expulser les 10000 familles de la zone et d'en accueillir 3500 dans des immeubles locatifs de 8 étages. L'éviction libérera environ 12 ha de terrain pour le développement de bâtiments commerciaux privés ou de résidences de luxe. 
ville, tous visant à créer un front de mer attractif et moderne, sans réelle considération des intérêts des classes populaires ${ }^{20}$.

Comme à Recife, l'intervention du secteur privé dans les projets d'urbanisme est de plus en plus marquée ${ }^{21}$. Celui-ci joue désormais un rôle prépondérant dans la création de nouveaux espaces. Le secteur public n'a plus qu'un simple rôle de "facilitateur ". Cette évolution participe à l'augmentation de la fragmentation (Mager, Matthey, 2012 : 4) (Simon, 1989 ; Balbo, 1993, cités par Balbo, 2002, p291).

En comparant les deux cas, on peut constater que, loin d'avoir résorbé la fragmentation socio-spatiale, les dynamiques liées à la globalisation exacerbent les inégalités et les dysfonctionnements sociaux, économiques, spatiaux et environnementaux au cœur de Recife comme de Chennai, même si l'émergence d'une classe moyenne témoigne des bénéfices de la croissance pour une partie toujours minoritaire de la population. La multiplication des échelles d'interventions et des acteurs impliqués dans les projets urbains a conduit à une complexification des processus décisionnels. La profusion d'acteurs complique les prises de décisions, renforce les conflits d'intérêt et crée une autre forme de fragmentation, en l'occurrence politico-institutionnelle. En somme, l'appel au secteur privé et la segmentation des processus décisionnels ont largement contribué à la déresponsabilisation et au désengagement de l'Etat dans la gestion métropolitaine, en tant que facteurs d'accroissement de la fragmentation urbaine.

Par ailleurs, le redéploiement des activités s'est accompagné de nouveaux modèles résidentiels, sous forme de condominios fechados au Brésil ou gated-communities en Inde. Ces quartiers fermés et équipés en infrastructures et en services représentent ainsi des « noyaux durs " au sein des villes en réservant l'accès au quartier à ses habitants. Particulièrement présent au Brésil, ce mode de résidence se généralise dans d'autres métropoles du « sud ». Une tendance similaire à l'enfermement est observée dans toutes les classes sociales, témoignant du sentiment d'insécurité et de l'entretien d'une peur

\footnotetext{
${ }^{20}$ Les cinq projets sont les suivants : la création d'un parc de restauration écologique, Adyar Poonga, réalisé par TNUIFSL, et décrié par les auteurs comme un projet de "green-washing" visant plutôt à embellir l'estuaire du fleuve Adyar ; le projet de voie express surélevée le long de la plage, dont la trajectoire se superposerait au quartier bidonville de Srinivasapuram; la proposition de construction d'un couloir de transport à grande vitesse le long du fleuve de l'Adyar ; la proposition de redéveloppement du quartier bidonville de Foreshore Estate et enfin le projet d'embellissement de la plage Marina.

${ }^{21}$ Un partenariat public-privé entre le gouvernement du Tamil Nadu et trois institutions financières - IL\&FS, ICICl and HDFC- permet au TNUIFSL de mobiliser d'importantes sommes pour mettre en œuvre des projets de renouvellement urbain à Chennai.
} 
réciproque. Cette peur de l'autre et les stratégies de repli sur soi et sur la communauté peuvent se lire notamment en réponse aux mutations de la société et comme autant de symptômes d'une crise sociale et identitaire.

Un réel paradoxe subsiste aujourd'hui entre le changement d'échelle et l'ouverture économique constatés dans les métropoles, et le repli social d'une partie de leurs populations, la construction de barrières physiques et l'isolement des communautés, qui restent les symptômes les plus visibles de la crise urbaine qui se pérennise dans un contexte mondialisé.

\section{Les contradictions des politiques urbaines face à la permanence de la fragmentation socio-spatiale}

Dans les deux villes s'exprime une importante mobilisation sociale, y compris au sein des appareils municipaux et des acteurs de la vie publique, en faveur de dispositifs visant à éviter l'expulsion des populations pauvres vers les périphéries extra-municipales. Les contestations ont émergé récemment en Inde, de façon discrète et isolée, impulsées notamment par des "élites", alors que les mouvements sociaux sont plus intégrés et répandus au sein de la société brésilienne.

A Recife, à la chute de la dictature, dès 1985, des études furent conduites par des chercheurs de la section d'urbanisme de la Faculté d'Architecture de l'Université Fédérale du Pernambouc en lien avec des urbanistes de la FIDEM, organisme public d'aménagement de la Région Métropolitaine, pour étudier la viabilité technique, urbanistique, juridique, et sociale du maintien de la population dans les zones d'habitat précaire proches des lieux d'emploi et de services, alors que parallèlement se structuraient des mouvements sociaux revendiquant le respect du « droit à la ville ». Cette conjonction explique l'adoption par la Municipalité de diverses mesures de requalification des favelas.

Plus tardivement, après l'accession du Président Lula au Pouvoir, les initiatives se sont multipliées en vue d'offrir des réponses au problème du logement des populations les moins solvables (Carrière, De la Mora, 2012). La loi fédérale portant création du «Estatuto da Cidade» (statut de la ville), et reconnaissant l'existence d'un "droit à la ville » pour tous, fut 
adoptée en 2001. En 2003, le Ministère fédéral des villes fut créé en vue de promouvoir des réformes visant une meilleure inclusion sociale des populations urbaines à faible revenu. Les Etats fédérés, à l'instar du Pernambouc, et les Municipalités ont aussi lancé des politiques spécifiques d'aménagement des favelas, en relais de celles de l'Etat, avec notamment la mise en place de budgets participatifs à destination des communautés faveladas. II s'agissait donc de créer des instruments favorisant une meilleure inclusion sociale des populations précaires, mais sans pour autant porter vraiment atteinte aux forces de marché toujours orientées vers la production immobilière de standing. Ainsi à Recife, jusqu'en 2012, le budget participatif était le plus important du pays entrant d'une certaine façon en concurrence avec les actions menées dans le cadre du PREZEIS (Programme de régularisation de zones spéciales d'intérêt social). Celui-ci est l'expression la plus directe de la lutte en faveur de la requalification des favelas et de la légalisation des occupations spontanées de terrains. Il fait suite à une première loi municipale, adoptée dès 1983, identifiant à Recife 29 quartiers répondant à des critères de précarité en leur conférant le statut de "Zones Spéciales d'Intérêt Social ». Le PREZEIS, en vue d'améliorer les conditions d'habitabilité, fit ensuite l'objet d'une seconde loi, en 1987. La loi de 1983 a institué au sein de chaque ZEIS, des structures de concertation avec les habitants : les COMUL. Elles sont supposées superviser le processus de régularisation foncière et associer les acteurs (habitants, ONG, représentants de l'Etat et de la municipalité...) au choix des travaux à entreprendre. Pour financer ceux-ci, fut institué le Fonds Municipal du PREZEIS, que le Municipe doit abonder chaque année à hauteur de 1,2\% des recettes fiscales municipales (Araujo et al. 1999). Cette ressource propre ne pouvant suffire, la majeure partie des projets est financée par le gouvernement fédéral, dans le cadre de coopérations entre l'Etat et le Municipe.

On dénombre aujourd'hui 67 ZEIS. Plus de $40 \%$ des habitants de Recife y résident, sur un peu moins de $12 \%$ de la superficie de la ville. En comparant ces deux pourcentages, on devine ce que peut être le degré de surpeuplement et de promiscuité dans les espaces concernés. Les ZEIS sont disséminées dans tout le territoire municipal, reflétant ainsi peu ou prou la géographie sociale de la ville, même si elles n'englobent pas toutes les zones $d^{\prime}$ habitat précaire (cf.Carte $n^{\circ} 7$ ). Les pressions visant à mettre en cause leur existence (Lucienne, 2014) restent fortes, notamment pour certaines offrant une localisation stratégique. 
La création des ZEIS et du PREZEIS ont ralenti le "boom » immobilier des 25 dernières années en mettant un frein à la réalisation de projets de moyen et haut standing à l'emplacement même des îlots précaires, mais sans parvenir à l'empêcher de façon définitive, et la question de l'alternative entre maintien sur place des populations pauvres avec requalification des favelas ou expulsions vers les lointaines périphéries après éradication reste toujours d'actualité (Lucienne, 2014). Qui plus est, le manque de foncier constructible pousse la municipalité à revenir parfois sur le principe du maintien sur place des populations pauvres en ouvrant à l'urbanisation les ZEIS offrant des avantages de localisation.

Dans son principe initial, le PREZEIS procède de la volonté de faire face à la gravité extrême de la précarité de l'habitat, d'associer les populations pauvres à la recherche d'une solution effective, et de contribuer à l'amélioration de la qualité de vie des communautés locales selon trois orientations :

- Résoudre la question de la propriété foncière (mais à ce jour la régularisation n'a été achevée dans aucune des ZEIS).

- Reconnaître le droit à la participation des habitants dans la définition des politiques urbaines au sein des périmètres concernés.

- Promouvoir de meilleures conditions matérielles de vie, par l'assainissement et l'aménagement de l'espace public.

Au total, 27 ans après le lancement du PREZEIS, 512.000 habitants (30\% de la population) ne sont plus aujourd'hui théoriquement menacés d'expulsion, mais le maintien sur place n'est en rien garanti de façon définitive. Des améliorations sont tangibles. Pour autant, le cadre territorial étroit de l'action municipale limite la portée du programme (De la Mora, 2012) dont le bilan paraît mitigé : Le problème crucial du " mal logement », et plus largement de la fragmentation socio-spatiale n'est pas résolu. Le PREZEIS n'est jamais parvenu à s'affirmer comme un fort instrument d'allocation des ressources municipales en faveur du logement des plus pauvres. En outre, le fonctionnement interne des ZEIS est loin d'être toujours optimal, du fait d'un manque d'organisation et de concertation entre les acteurs, et probablement d'un manque de préparation et de formation des représentants des habitants. Dépendantes de concours fédéraux, les ressources dédiées à la lutte contre la fragmentation urbaine ne sont pas à la hauteur des enjeux. Pour toutes ces raisons, l'impact 
d'une politique de requalification et d'amélioration de l'habitabilité des favelas, telle que celle de Recife, reste modeste et insuffisant.

A Chennai, la résorption de l'habitat précaire a suivi une voie certes différente, mais pas plus efficiente. Les premiers programmes de requalification des bidonvilles sont apparus tardivement, à partir des années 1960, et les actions ont principalement consisté à améliorer ponctuellement les conditions d'habitabilité par la réalisation d'infrastructures de base, le renfort des structures des habitations ou l'offre de matériel électroménager. Ces mesures, guidées par des intérêts politiques, ont largement contribué à la politisation des bidonvilles et à l'instauration d'un rapport clientéliste entre l'Etat et les habitants. En outre, le caractère à la fois local et "court-termiste" de ce type d'interventions a bridé le développement d'une réflexion globale sur les possibles stratégies d'intégration urbaine des bidonvilles.

Un service de l'Etat traitant spécifiquement de la requalification et/ou de l'élimination des bidonvilles a été créé en 1971, le Tamil Nadu Slum Clearance Board (TNSCB) a été chargé de localiser les zones d'habitat précaire dans le tissu urbain et de mettre en œuvre des programmes de redéveloppement ou de relogement. L'Etat lui a alors assigné trois priorités stratégiques : le développement in-situ, la reconstruction in situ et enfin le relogement. Nithya Raman (2011) note que les premières actions du TNSCB ont été déterminées par des priorités politiques, favorisant la reconstruction des bidonvilles sur place, et montre comment cette dynamique s'est modifiée avec l'entrée de la banque mondiale en 1975 dans le financement du secteur urbain, contribuant à dissocier le TNSCB des influences politiques. A la fin des années 1980, les politiques urbaines en Inde ont reflété un changement majeur du rôle de l'Etat, passant du statut de fournisseur de logements à celui de facilitateur, à travers la promotion de partenariats publics-privés (Raman, 2011). Les années 2000 ont ensuite marqué un tournant décisif dans l'approche du gouvernement envers le bidonville: une succession de plans de renouvellement urbain ont été mis en œuvre pour faire face au problème de la précarité résidentielle, en donnant la priorité au relogement des habitants en périphérie. Après le projet pilote de modernisation urbaine JNNURM $^{22}$ mis en œuvre de 2005 à 2013, le Plan national de renouvellement urbain Rajiv Awas Yojana, de 2013 à 2015,

\footnotetext{
22 Jawaharlal Nehru National Urban Renewal Mission
} 
(aujourd'hui " Housing for All $»^{23}$ ) poursuit l'objectif de « villes sans bidonvilles » et prévoit le redéveloppement des zones précaires dans leur intégralité par le relogement de ses habitants. Ce plan d'action comprend deux parties, s'articulant dans une approche plus globale que les précédentes stratégies : l'amélioration ou le relogement des bidonvilles existants accompagné de l'octroi de droits de propriété ; ainsi que des actions de prévention d'apparition de nouveaux quartiers précaires, intégrant la réservation de terrains et de logements à destination des populations pauvres. Mais les conditions de mise en œuvre ne dépassent pas le stade des bonnes intentions; de grands programmes de relogement en périphérie ont été engagés, à une vingtaine de kilomètres de la ville : des habitants de 78 zones urbaines précaires différentes ont été relogés sur les deux principaux sites de Kannagi Nagar et Semmenchery. Au total, 22390 immeubles y ont été construits accueillant 103774 personnes ${ }^{24}$.

Le succès de ces programmes est pour le moins controversé : des rapports d'études indiquent qu'à Kannagi Nagar, 20 \% des appartements seraient vacants et 50\% ne seraient plus occupés par les bénéficiaires d'origine (Raman, Narayan, 2013). Malgré ces déficiences apparentes et la montée de problèmes sociaux liés à un phénomène de "ghettoïsation » (Coelho et al., 2012), le TNSCB s'est appuyé sur cet exemple pour reproduire un troisième site de relogement à Perumbakkam, à 6 kilomètres au sud du premier. Réalisé sous le programme JNNURM, ce site prévoit d'accueillir 20000 familles relogées sous différents programmes de renouvellement urbain ${ }^{25}$.

Les discours affichés en faveur de l'intégration des zones d'habitat précaire dans le tissu urbain se voient contredits à l'épreuve des faits: dans la pratique, les projets d'envergure métropolitaine sont retenus en priorité et justifient un relogement massif des habitants de bidonvilles en périphérie, ce qui renforce la ségrégation urbaine et perpétue les mécanismes d'exclusion. Si le phénomène de mondialisation a favorisé des avancées en ce qui concerne les droits individuels, des concepts tels que le "droit à la ville » ou au logement semblent se

\footnotetext{
${ }^{23}$ Depuis 2015, le progamme Pradhan Mantri Awas Yojana, aussi appelé « Housing for All » a remplacé le plan Rajiv Awas Yojana. Ce nouveau programme, en vigueur de 2015 à 2022, priorise le redéveloppement des quartiers précaires sur place.

${ }^{24}$ D'après le rapport du People's Union for Civil Liberties (PUCL), "Fact Finding Team on Forced Eviction and Rehabilitation of Slums Dwellers in Chennai". Fact Finding Report - Tamil Nadu and Puducherry, 2010.

${ }^{25}$ Kannagi Nagar a en premier lieu été construit pour réhabiliter les habitants affectés par le tsunami de 2004, puis progressivement l'ensemble des sites ont accueilli les résidents de bidonvilles évincés par la mise en œuvre de projets de redéveloppement des voies fluviales, de projets de restauration environnementale ou dans le cadre des mesures post-inondations de décembre 2015.
} 
concrétiser difficilement dans le contexte indien. La réflexion peine à s'enclencher sur le devenir des zones de précarité, et la mobilisation de la société civile reste faible au regard des dynamiques urbaines à l'œuvre.

Au total, au Brésil comme en Inde, on retrouve dans les politiques publiques une volonté de traiter les zones de précarité par une amélioration des conditions d'habitabilité et une meilleure insertion urbaine. Néanmoins, l'observation révèle des résultats très partiels, voire antinomiques avec les objectifs affichés, alors que de réelles menaces pèsent sur le respect du droit à la ville sous la pression du marché.

Dans les deux cas, le défi de l'insertion socio-spatiale des populations en grande précarité renvoie à un autre dilemme entre éradication ou requalification des espaces de précarité, avec pour enjeu ultime la garantie du « droit à la ville ».

Au Brésil, une nouvelle réflexion sur l'intégration et la requalification des favelas dans le territoire urbain émerge. La ville de Recife se distingue certes par son rôle de pionnier dans I'institutionnalisation des ZEIS et la régularisation foncière des zones d'habitat précaire, mais cette orientation stratégique reste soumise à des pressions de plus en plus fortes en faveur de politiques impliquant au contraire l'éradication des favelas et le transfert de leurs habitants en lointaine périphérie, en vue de libérer du foncier pour des opérations immobilières de grande envergure. En revanche, à Chennai, la stratégie de l'éradication et du relogement l'a emporté de façon décisive. Malgré les discours en faveur de l'insertion des quartiers précaires, le "déguerpissement " avec relogement reste la pratique la plus courante. Dans ce processus, la puissance publique adopte des postures contradictoires, étant tiraillée entre un objectif de compétitivité et d'attractivité économique de la ville et le souci de prendre en compte les demandes d'urbanisation des quartiers précaires formulées par leurs habitants. L'oscillation entre le rejet et la reconnaissance des bidonvilles reflète alors les différentes visions portées sur les zones d'habitat précaire et sur l'espace urbain. Dès lors, deux échelles de planification urbaine coexistent et entrent en conflit : l'échelle stratégique qui défend la valeur marchande du sol urbain et la mise en œuvre de grands projets d'envergure mondiale, et, l'échelle participative des populations pauvres à travers divers dispositifs destinés à améliorer la qualité de vie dans leurs espaces (Melo, 2013).

Au total, Chennai et Recife nous montrent qu'un développement urbain durable ne pourra pas se concrétiser sans une réflexion plus globale sur les modalités de la résorption de la fragmentation socio-spatiale dans les métropoles du « sud ». 
Le paradoxe des politiques publiques brésiliennes est finalement de maintenir cette fragmentation spatiale de manière volontaire à travers la mise en place des ZEIS, en vue d'une intégration sociale des populations des favelas. L'action publique en direction des favelas entre de plus en plus en contradiction avec la volonté de « fabriquer une ville visible et attractive "... II faut alors s'interroger sur les leviers d'action à mobiliser pour résorber à la fois les disparités sociales et l'habitat précaire, ce qui pose la question d'une action combinée, associant décideurs publics et société civile.

\section{Conclusion}

Nos deux cas d'étude nous amènent à des conclusions aisément transposables à d'autres contextes métropolitains du "sud " en nous donnant à voir un niveau de fragmentation socio-spatiale encore très important. A cause de cela, à Recife, comme à Chennai, les conditions ne sont pas réunies pour un développement urbain socialement inclusif à l'échelle de la métropole dans son ensemble. Au cœur de tensions dialectiques entre paradigme de l'attractivité et insertion socio-spatiale, les pouvoirs publics affichent des stratégies contradictoires, et, les projets urbains mis en œuvre sont révélateurs de conflits se manifestant à différents niveaux: des conflits d'intérêts, d'échelles mais aussi de temporalités, renvoyant à différents degrés de planification et de visions portées sur le territoire. Par ailleurs, le changement d'échelle lié à la mondialisation soulève de plus en plus des questions identitaires suscitées par la coexistence de modèles et valeurs " traditionnels » et de concepts issus des sociétés occidentales. Ainsi, avec la globalisation, c'est non seulement le rapport à l'espace qui est revisité avec une « réduction des distances ", mais aussi le rapport au temps et aux individus. Les profondes mutations que connaissent les sociétés des pays émergents suscitent l'apport d'une nouvelle réflexion générale sur l'articulation de la dialectique local/global et demandent à repenser l'échelle du territoire, ainsi que la définition même de la ville. 


\section{Bibliographie}

AGIER, Michel (1999) L'invention de la ville. Banlieue, township, invasions et favelas. Editions des archives contemporaines, 176 pages

Alves dos SANTOS, O. - BARbosa MAHMOOD, S. (2013) Para pensar geograficamente a " pobreza urbana »: o sistema de informações georeferenciadas dos assentamentos populares da Região metropolitana do Recife/PE. Communication au XIIle simposio nacional de geografia urbana, UERJ, Rio de Janeiro, $18-22$ de novembro 2013, 19 pages.

ARABINDOO, Pushpa (2009) Falling Apart at the margins ? Neighbourhood Transformations in Peri-urban Chennai. Development and Change. September 2009, Vol. $40 \mathrm{n}^{\circ}$ 5,pp. 879 - 901. DOI 10.1111/J.14677660.2009.01587.

ARABINDOO, Pushpa, 2012. The spatial (il)logic of slum resettlement sites in Chennai, presenté à la conference "Speculating on Slums" au Départment de Géographie de Royal Holloway University of London. 23/05 2012.

ARAUJO, Antonio. MARINHO G. (1999) Uma politica inovadora de urbanização no Recife, 10 anos de PREZEIS. Ed : CJC, ETAPAS e FASE

BITTENCOURT SANTOS Breno (2015) Impactos da organização social do território nas condições de vida urbana: uma análise do Índice de Bem-Estar Urbano Local na Região Metropolitana do Recife, in DE ALMEIDA SOUZA M.A. et BITOUN J. (editores) (2015) RECIFE: Transformações na ordem urbana, Observatório das metropoles, chap. 9 pp. $348-389$.

BRETAGNOLLE Anne, LE GOIX Renaud, VACCHIANI-MARCUZZO Céline (2011) Métropoles et mondialisation. La documentation française, Paris, 64 pages

CARRIERE Jean-Paul (2012) Regards croisés sur les dynamiques métropolitaines au Brésil et en Europe, in L-N. TELLIER (ed.) Métropoles des Amériques en mutation, Presses Universitaires du Québec, pp. 31 - 46

CARRIERE Jean-Paul. - DE LA MORA Luis (2012) Regards croisés France-Brésil sur les processus et enjeux de la métropolisation au Brésil. Communication aux « dialogues France - Brésil » APERAU - ANPUR - São Paulo, 27 30 mars 2012 - 20 pages

CARRIERE Jean-Paul - DE LA MORA Luis (2014) Précarité urbaine et fragmentation socio-spatiale au sein des métropoles brésiliennes : le cas de Recife, Revue Géographie, Economie, Société, № 4 pages 373 - 397

CARY Paul. (2010) «Réflexions sur la société de consommation brésilienne " Problèmes d’Amérique latine, 78, Pages 75-90

CHETRY Mickaël (2010) « Les habitants des favelas face au droit à la ville au Brésil : réalité de la fragmentation urbaine, défi de l'intégration ", Doctorat de géographie- Aménagement. Université Jean Moulin, Lyon III

CHETRY Mickaël. (2013) " La fragmentation, un nouveau regard sur la ville brésilienne ? » in CARREL M. CARY P. WACHSBERGER J-M. (dir.) Ségrégation et fragmentation dans les métropoles. Perspectives internationales, pp. $121-136$.

COELHO Karen, VENKAT T, CHANDRIKA R (2012) The spatial reproduction of urban poverty: labour and livelihoods in a slum resettlement colony, in economic and political weekly (review of urban affairs), vol xlvii, nos 47 and 48, december 2012

DE ALMEIDA SOUZA M.A. et BITOUN J. - editores - (2015) RECIFE: Transformações na ordem urbana, Observatório das metropoles, INCT et Letra Capital editora, 538 pages

DE ALMEIDA SOUZA Maria Angela, CORTÊS DE LIMA Rosa Maria, LIRA DOS ANJOS Kainara, BARBOSA DA SILVA Ailson (2015) As formas de provisão da moradia na configuração socioespacial da Região Metropolitana do Recife, in DE ALMEIDA SOUZA M.A. et BITOUN J. (editores) (2015) RECIFE: Transformações na ordem urbana, Observatório das metropoles, chap. 6 pp. $241-285$

DE LA MORA, Luis (2011) Gatted Communities in shanty towns and governmental housing programs. $6^{\text {th }}$. INTERNATIONAL CONFERENCE OF THE RESEARCH NETWORK "Private Urban Governance \& Gated 
Communities" on "Re-Interpretation /Transformation of Territorial Boundaries" ISTAMBUL 08 - 11 September 2011, 14 pages

HOCHART, Karine (2014) Lecture croisée de dynamiques urbaines en Inde et au Brésil. Habitat informel et fragmentation socio-spatiale à Chennai et Recife. Rapport de fin d'études, Département Aménagement, Polytech'Tours, 92 pages

KENNEDY, Loraine, VARREL, Aurélie, DENIS, Eric, DUPONT, Véronique, DHANALAKSHMI, R., ROUMEAU, Samuel, BAUD, Isa, PFEFFER, Karin, SRIDHARAN, N., VIJAYABASKAR, M., SURESH BABU, M., SEIFELISLAM, Aicha, ROUANET, Hortense et SAHARAN, Tara (2014) 5: Engaging with Sustainability Issues in Metropolitan Chennai. Chance 2 Sustain.

LUCIENNE Cynthia (2014) Les défis de l'insertion socio-spatiale des favelas à Recife: entre exigences d'attractivité et nécessités d'habitabilité. Le cas de la ZEIS du Coque. Thèse de Doctorat en Aménagement de l'espace et urbanisme, Université de Tours et Universidade Federal do Pernambouc, 279 pages

MAGER, Christophe, MATTHEY, Laurent (2012) " Entre fragmentation et ordre urbain: une géographie politique des espaces de l'entre-soi ». L'espace politique, 17: 1-7.

MARIUS-GNANOU,Kamala (2010) « Nouvelles actiivtés économiques et dynamique métropolitaine : le cas de la périphérie sud de Chennai » Annales de géographie, 2010, Vol. 671-672,pp.28. DOI 10.3917/ag.671.0028

MUTHIAH, Subbiah (2008) Madras rediscovered, A historical guide to looking around, supplemented with tales of „Once Upon a City“, East West, Chennai

MELO Anna-Maria. (2013) «Recettes identiques, impacts contrastés : la planification stratégique à Lille et à Recife »" in CARREL Marion. CARY Paul. WACHSBERGER Jean-Michel. (dir) "Ségrégation et fragmentation dans les métropoles. Perspectives internationales » pp. 217 - 240

NAVEZ-BOUCHANINE Françoise (2002) La fragmentation : sources et définitions, in NAVEZ-BOUCHANINE (dir.) La fragmentation des villes en question : des villes entre fragmentation spatiale et fragmentation spatiale ? Paris, L'Harmattan, p. $33-67$

NINNIN Justine. (2014) Territorialisation et nouveaux modes d'action de sécurisation et de planification urbaine : une innovation sociale à Rio de Janeiro, colloque Attilio, Lille, 24 - 25 septembre 2014

OLIVEAU, Sébastien (2007) "Etalement urbain et fragmentation: Chennai, un exemple en Inde du Sud ", Manuscrit auteur, publié dans Etalement urbain et ville fragmentée dans le monde, des théories aux faits, Orléans: France (2007)" halshs-00186654, version 1 - 10 Nov 2007

RAMAN, Nithya (2011) "The Board and the Bank: Changing Policies towards Slums in Chennai." Economic \& Political Weekly, July 2011, vol. XLVI, n³1, pp. 74 - 80

RAMAN Nithya V., NARAYAN Priti, (2013) "India's invisible population", The Hindu, published the 19/10/13: http://www.thehindu.com/opinion/lead/indias-invisible-population/article5248725.ece, visited the 06/03/14

SANTOS Milton (1982) Ensaios sobre a urbanização Latino-Americana. São Paulo: Editora Hucitec,

SARAVANAN, K. JAYARAMAN N. (2011). Robbing the poor: slum housing and the elite environmentalism of Adyar Poonga [en ligne]. Save Chennai beaches campaign. http://www.sipcotcuddalore.com/downloads/Rob the poor.pdf

SASSEN, Saskia. (1991) : la ville globale New York, Londres, Tokyo, ed Descartes et Cie, Paris, 530 pages

VAGUET, Odette (1997) "La ville indienne: un espace fragmenté », Le géographe et les frontières, éditions L'Harmattan

VALLADARES Lucia (2000) "Qu'est-ce qu'une favela? » Cahiers des Amériques Latines, (34), pp 61 - 72

VALLADARES Lucia (2006) La favela d'un siècle à l'autre, Editions de la Maison des sciences de l'homme, Paris Collection Horizons Américains, 229p.

VIDAL, Laurent (1994) " Les mots de la ville au Brésil. Un exemple: la notion de " fragmentation » Cahiers des Amériques Latines, $\mathrm{n}^{\circ} 18$, p. 161-181. 


\section{Annexe}
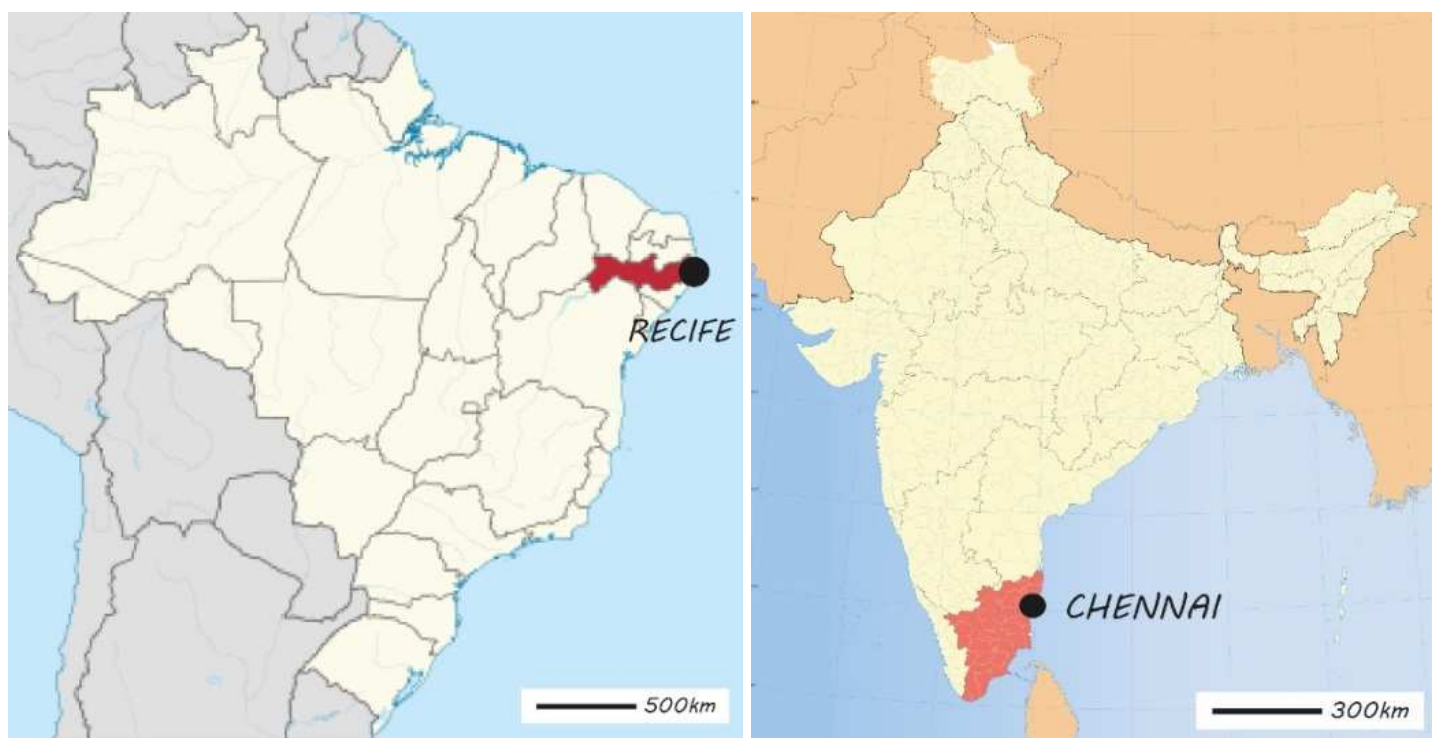

Cartes $n^{\circ} 1$ et $n^{\circ} 2$ : Localisations de Recife (Etat du Pernambouc) et de Chennai, (Etat du Tamil Nadu). Source : Hochart, 2015

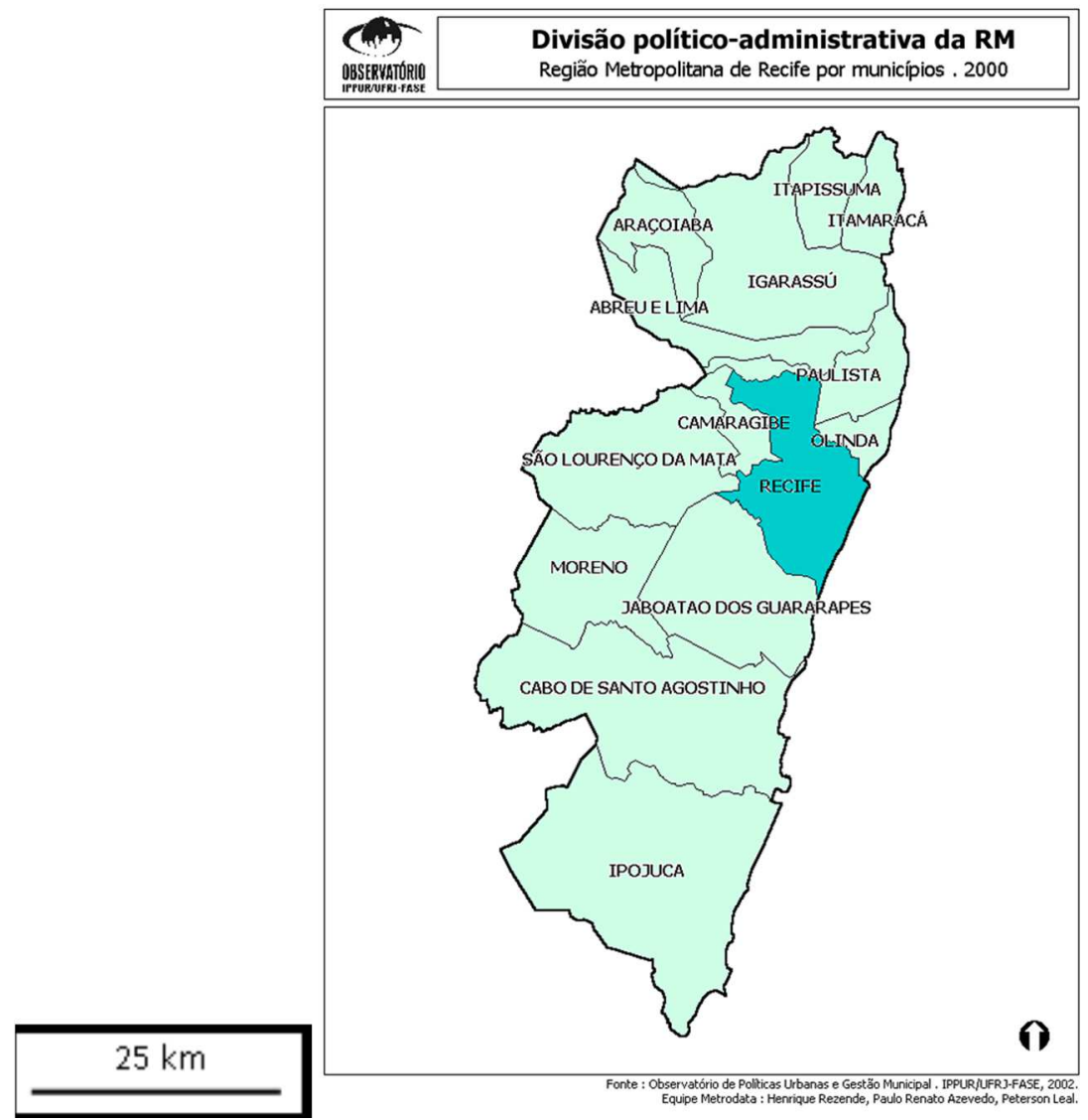

Carte $n^{\circ} 3$ : Région Métropolitaine de Recife.

Source : www.observatoriodasmetropoles.ufrj.br 


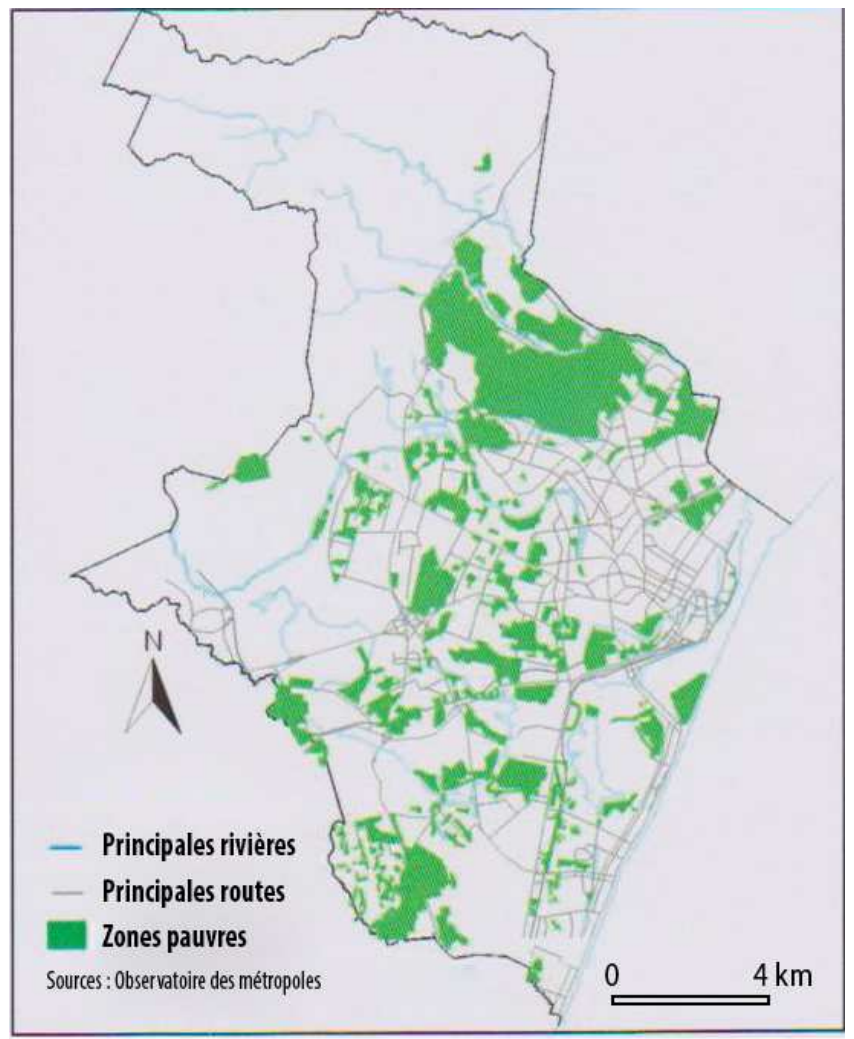

Carte $n^{\circ} 4$ : les zones d'habitat précaire à Recife. Source : Observatório das Metrópoles, Recife. (2005) - traitement : Dominique Andrieux, UMR CITERES, Tours

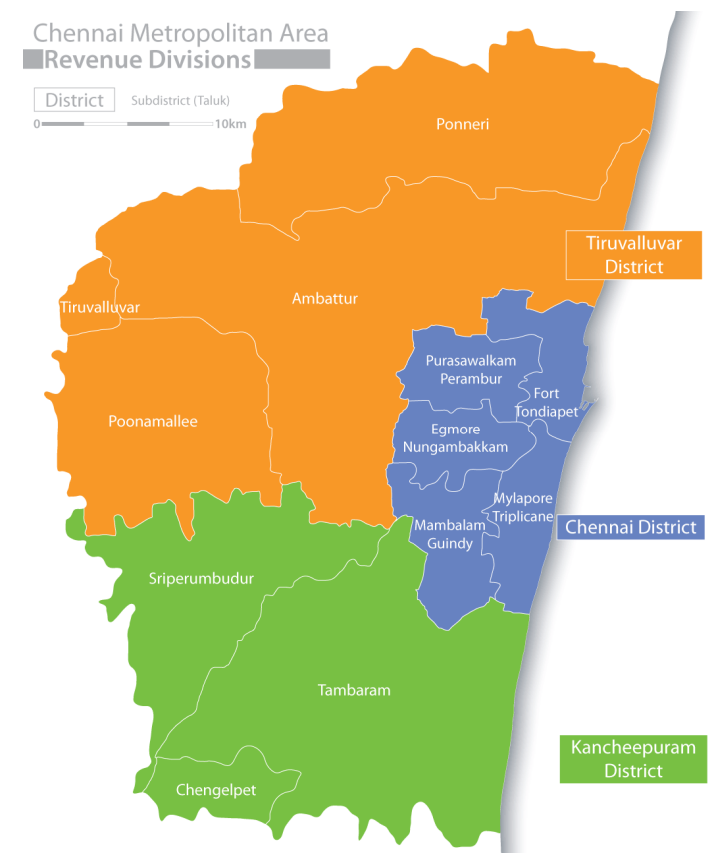

Carte $n^{\circ} 5$ : Région métropolitaine de Chennai. Source : de.academic.ru/dic.nsf/dewiki/252393

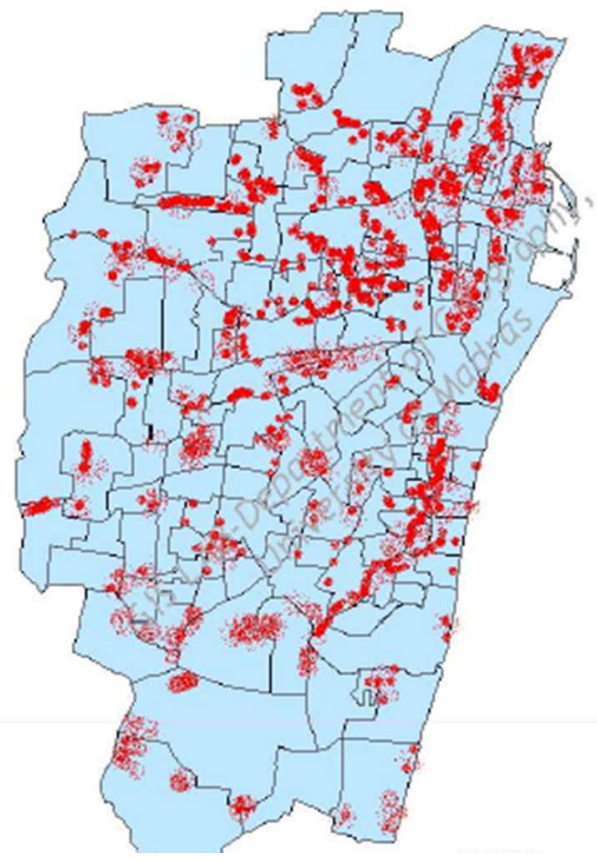

Carte $n^{\circ} 6$ : Les zones d'habitat précaire dispersées dans le tissu urbain à Chennai.

Source: R. Jaganathan et G. Bhaskaran, 2008 

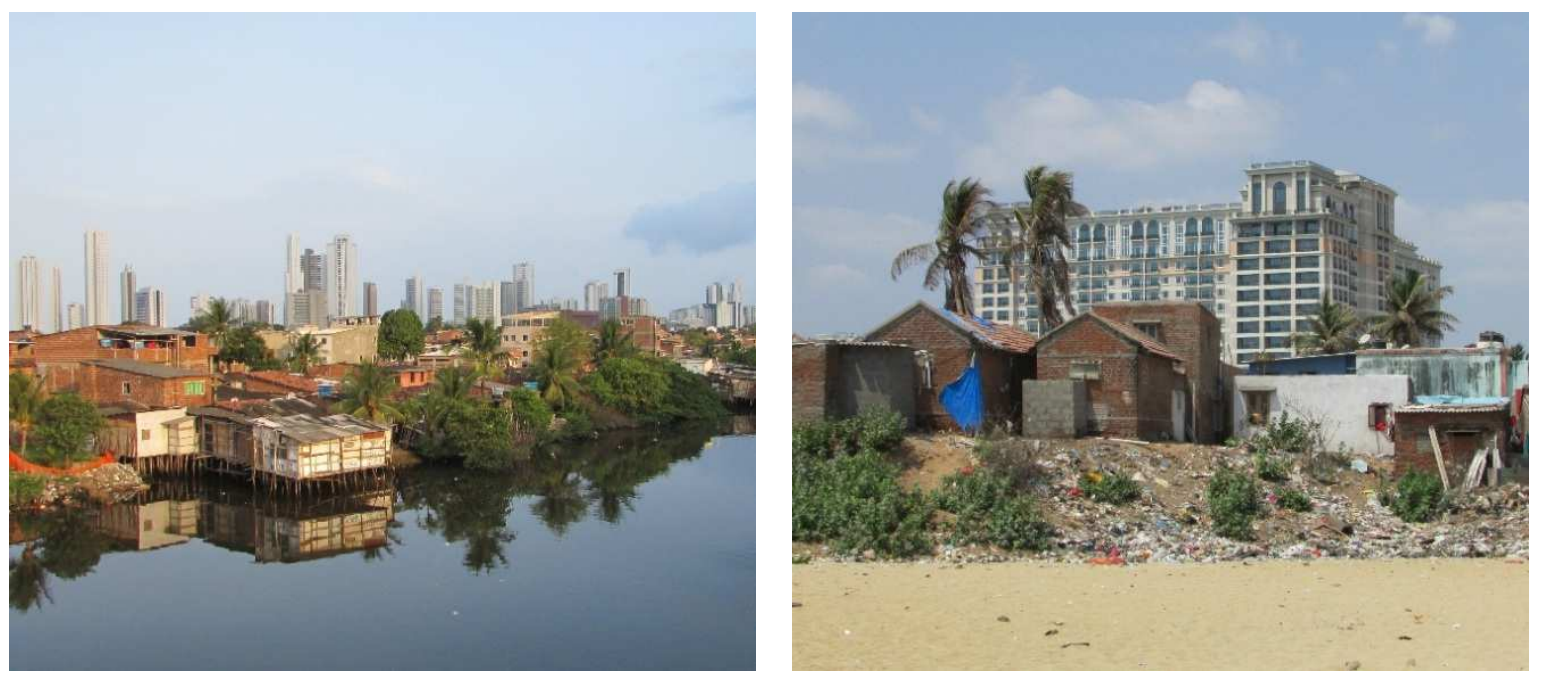

Photographies $n^{\circ} 1$ et $n^{\circ} 2$ : Coexistence de zones d'habitat précaires et d'immeubles de standing, à Recife (gauche) et à Chennai (droite). Source : Hochart, 2014

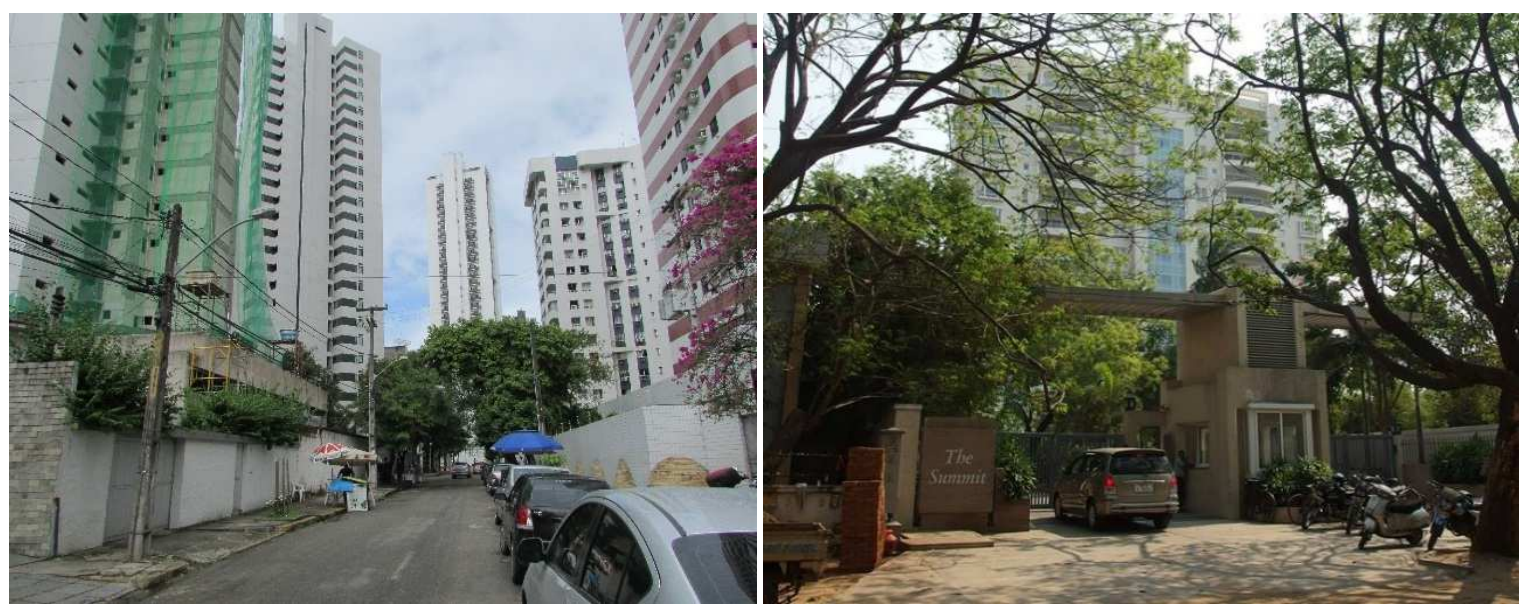

Photographies $n^{\circ} 3$ et 4 : Copropriétés multifamiliales à accès limité et contrôlé dans le quartier de Boa Viagem, Recife (à gauche) et dans le quartier Adyar à Chennai (à droite).

Source : Hochart, 2014. 


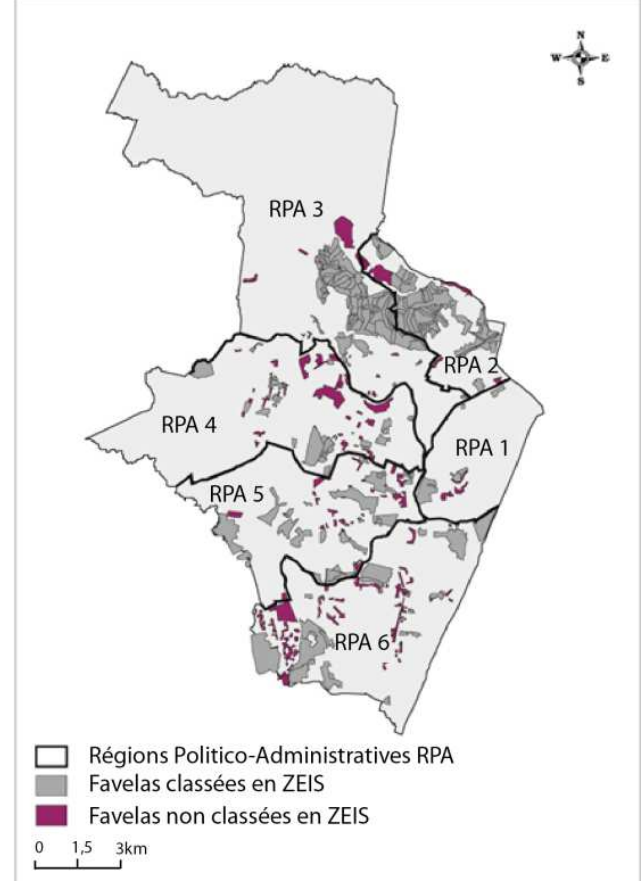

Carte $n^{\circ} 7$ : Favelas et ZEIS de Recife. Source: Observatório de Políticas Públicas - PE, 2005 / UFPE, FASE - PE. Traitement : Michaël Chétry, 2010
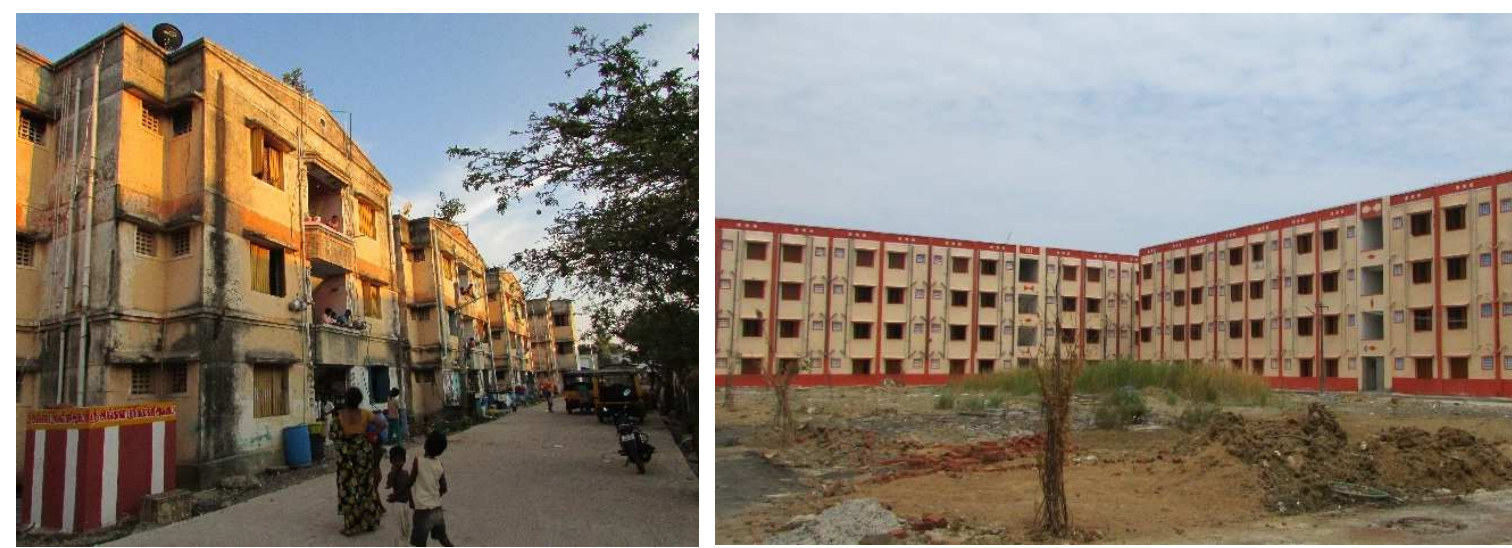

Photographies $n^{\circ} 5$ et 6 : Bâtiments de relogement à Kannagi Nagar, périphérie sud de Chennai. Source : Hochart, 2014 